\title{
Impact of extracellular matrix derived from osteoarthritis subchondral bone osteoblasts on osteocytes: role of integrin $\beta 1$ and focal adhesion kinase signaling cues
}

\author{
Indira Prasadam ${ }^{1 *}$, Saba Farnaghi ${ }^{1}$, Jian Q Feng ${ }^{3}$, Wenyi Gu', Samuel Perry', Ross Crawford ${ }^{1,2}$ and Yin Xiao ${ }^{1}$
}

\begin{abstract}
Introduction: Our recent study indicated that subchondral bone pathogenesis in osteoarthritis $(\mathrm{OA})$ is associated with osteocyte morphology and phenotypic abnormalities. However, the mechanism underlying this abnormality needs to be identified. In this study we investigated the effect of extracellular matrix (ECM) produced from normal and $\mathrm{OA}$ bone on osteocytic cells function.

Methods: De-cellularized matrices, resembling the bone provisional ECM secreted from primary human subchondral bone osteoblasts (SBOs) of normal and OA patients were used as a model to study the effect on osteocytic cells. Osteocytic cells (MLOY4 osteocyte cell line) cultured on normal and OA derived ECMs were analyzed by confocal microscopy, scanning electron microscopy (SEM), cell attachment assays, zymography, apoptosis assays, qRT-PCR and western blotting. The role of integrin $\beta 1$ and focal adhesion kinase (FAK) signaling pathways during these interactions were monitored using appropriate blocking antibodies.

Results: The ECM produced by OA SBOs contained less mineral content, showed altered organization of matrix proteins and matrix structure compared with the matrices produced by normal SBOs. Culture of osteocytic cells on these defective OA ECM resulted in a decrease of integrin $\beta 1$ expression and the de-activation of FAK cell signaling pathway, which subsequently affected the initial osteocytic cell's attachment and functions including morphological abnormalities of cytoskeletal structures, focal adhesions, increased apoptosis, altered osteocyte specific gene expression and increased Matrix metalloproteinases (MMP-2) and -9 expression.
\end{abstract}

Conclusion: This study provides new insights in understanding how altered OA bone matrix can lead to the abnormal osteocyte phenotypic changes, which is typical in OA pathogenesis.

\section{Introduction}

Bone matrix serves as an organized framework for bone as a tissue, offering mechanical support and mediating biological activities of bone cells and signals that maintain bone homeostasis and remodelling [1]. Bone cells, like most other matrix-associated cells, cannot survive or differentiate without adhesion to their matrix [2,3]. Consequently, bone cell morphology and functions can depend strongly on matrix quality under conditions in which biological signals are constant. In osteoarthritis

\footnotetext{
* Correspondence: i.prasadam@qut.edu.au

${ }^{1}$ Institute of Health and Biomedical Innovation, Queensland University of Technology, Kelvin Grove Campus, Brisbane, Queensland 4059, Australia Full list of author information is available at the end of the article
}

(OA) it is well-known that subchondral bone matrix, structure, organisation, composition and mineralisation are abnormal when compared to normal bone [4].

Osteocytes are the most abundant and longestliving cells in the adult skeleton. The importance of osteocytes in regulating bone remodeling and turnover has been generally acknowledged [5]. Our recent study demonstrated that various functional and morphological properties of osteocytes appear to be hampered in patients with OA, indicating that these cells could play an important pathological role in subchondral bone sclerosis [6]. However, the potential molecular mechanism behind this abnormal 
osteocyte behaviour in OA patients is yet to be identified.

In vivo, osteocyte cells under normal conditions, contact a complex mixture of secreted 'extracellular matrix' (ECM) proteins called the bone matrix. The bone matrix isolates osteocytes from each other and instead osteocytes interact with other osteocytes and other bone cells by an elaborate network of osteocytes (dendritic) processes. The contact with the bone matrix is a critical mechanism providing cues via cytoplasmic processes called canalicules to form a cellular network to sense efficiently both mechanical and systemic stimuli [7]. On the other hand, it seems that osteocytes which become transformed in diseases such as osteoporosis and OA are characterised by loose contact with ECM substrate leading to morphological and functional bony changes $[6,8]$. Primarily based on our previous observations, in this study we hypothesised that altered mineralisation and the ECM quality of the subchondral bone matrix is the trigger for the osteocyte abnormalities seen in OA.

In vivo cell adhesion to the ECM is mediated by integrin $\beta 1$ receptors. Bone ECMs are composed of several macromolecules including fibronectin, laminin, collagens and proteoglycans. A number of these ECM proteins contain the three amino acid sequence ArgGly-Asp (RGD), which is exclusively recognised by corresponding integrin $\beta 1$ receptors $[9,10]$. Attachment of integrins with the above macromolecules can activate the downstream signalling focal adhesion kinase (FAK) and vinculin that can initiate a cascade of phosphorylation events that fine-tune cell-type-specific phenotypes [11]. Maintenance of integrin linkages is essential for cell adhesion, proper cytoskeletal organisation and function of the specific cell types. It has been demonstrated previously that disruption of these attachments, via addition of neutralising antibodies or peptides, can induce cells to detach from the ECM resulting in apoptosis, structural alterations and cellular dysfunction. The aim of this study is to test how normal and OA bone ECM differentially regulates the function of the osteocytes. The other objective of the present study is to reveal the critical role of cell-matrix adhesions governing this process, notably involving the integrin $\beta 1$-FAK signalling axis.

\section{Methods}

\section{Subchondral bone osteoblast (SBO) isolation and characterisation}

The Ethics Committee of Queensland University of Technology and the Prince Charles Hospital approved this study and the participants' written consent was obtained according to the Declaration of Helsinki (Ethics Number: 0700000157). Knee bone specimens were taken within $5 \mathrm{~mm}$ of the subchondral bone plate as described previously in our studies [12-14]. OA SBOs were cultured from bone sourced from the medial compartment of the knee from patients suffering advanced OA, where the cartilage was degraded and showed prominent subchondral bone sclerosis and density $(n=5)$ (age: $61.5 \pm 4$ years). Normal SBOs were cultured from bone collected from trauma patients who were undergoing above the knee amputations with no evidence of subchondral bone sclerosis or cartilage degeneration on top of it $(n=4)$ (age: $60.1 \pm 6$ years). The criteria for the OA diagnosis were those established according to the American College of Rheumatology [15]. None of the normal patients had any musculoskeletal disorders, such as osteoporosis. SBOs were isolated according to the method described by Beresford [16,17]. Isolated normal and OA SBOs were characterised for their phenotype as described in our previous studies [12,13]. Passage one SBOs were used for this study.

\section{Subchondral bone osteoblast differentiation and preparation of de-cellularised matrices}

Osteogenic differentiation of normal and OA SBOs (20,000 cells per well) was performed in (D)MEM medium containing osteogenic supplements (10 nM dexamethasone, $10 \mathrm{mM} \beta$-glycero-phosphate, $50 \mu \mathrm{g} / \mathrm{mL}$ ascorbic acid) on coverslips (NUNC, Roskilde, Denmark) (placed on 24 well plates) for five weeks. After five weeks, SBOs were rinsed two times in $1 \mathrm{X}$ PBS. Next, to produce the de-cellularised matrices, $0.02 \mathrm{M}$ ammonium hydroxide in $\mathrm{ddH}_{2} \mathrm{O}$ was applied for 20 to 30 minutes at room temperature intermittently visualising under a light microscope for cell roundup and lysis. Then ammonium hydroxide was removed by inverting the culture surface, with plates washed several times with sterile $1 \mathrm{X}$ PBS before seeding osteocytes on top of them.

\section{Characterisation of de-cellularised matrices}

The removal of cells and the retention of a collagenous matrix were verified following de-cellularisation. Briefly, cells were fixed in $4 \%$ paraformaldehyde for ten minutes, washed three times with 1X PBS and permeabilised with $0.1 \%$ Triton for five minutes and stained with ProLong Gold Antifade Reagent (Invitrogen, Life Technologies Australia Pty Ltd, Victoria, Australia) with 4,6-diamidino-2phenylindole (DAPI) on a glass slide. The absence of nuclei was confirmed using fluorescence microscopy (Zeiss, using AxioVision Image analysis software). The presence of collagen I and various ECM proteins in the de-cellularised matrices was confirmed by immunostaining and western blotting following the protocol described in our previous studies $[12,13]$. The presence of mineral content was confirmed by staining matrices with $1 \%$ alizarin red. Scanning electron microscopy (SEM) with energy dispersive X-ray analysis (SEM/ EDX) (FEI Quanta 200 Environmental SEM equipped with an Evarhart Thomley secondary electron detector) was used 
to examine the morphology of the calcium phosphate deposit and to obtain the elemental composition in normal and OA matrices.

\section{MLOY4 osteocyte cell line culture}

The well-characterised osteocytic line, MLOY4 (provided by Dr. Lynda Bonewald) [18] was cultured on T75 tissue culture flasks coated with type I collagen $(0.30 \mathrm{mg} / \mathrm{ml}$; Sigma, St. Louis, MO, USA) in osteocyte culture medium containing alpha modified essential medium ( $\alpha \mathrm{MEM}$; GIBCO BRL, Grand Island, NY, USA), 5\% fetal bovine serum (FBS; Hyclone, Logan, UT, USA), 5\% calf serum (CS), and $1 \%$ penicillin and streptomycin (GIBCO BRL).

\section{Seeding MLOY4 cells on normal and osteoarthritis matrices}

MLOY4 cells (10,000 cells/cover slip) were seeded directly on the top of the normal and OA matrices prepared above and incubated in osteocyte medium at $37^{\circ} \mathrm{C}$ containing $5 \%$ $\mathrm{CO}_{2} / 95 \%$ atmospheric air at different time points.

\section{Integrin and focal adhesion kinase signalling studies}

To confirm the integrin $\beta 1$ mediated molecular mechanism, MLOY4 cells were cultured on normal and OA matrices in the presence or absence of blocking-integrin $\beta 1$ $(10 \mu \mathrm{m})$ antibody (Clone P5D2, Chemicon, Millipore, MERCK Pvt Ltd, Victoria, Australia) or with an irrelevant antibody. P5D2 react with the human $\beta_{1}$ integrin subunit and block adhesion of cells to ECM proteins. FAK activation and de-activation was analysed by anti-phospho- FAK and anti-total FAK antibodies (Cell Signaling Technology, Gene search Pty Ltd, Queensland, Australia).

\section{Scanning electron microscopy}

The morphology of osteocytes grown on normal and OA osteoblast matrices were assessed by SEM as described previously [19]. (+/- MLOY4 cells) were fixed with a solution containing $3 \%(\mathrm{v} / \mathrm{v})$ glutaraldehyde in $0.1 \mathrm{M}$ sodium cacodylate buffer solution $\left(\mathrm{pH} \mathrm{7.3)}\right.$ for one hour at $4^{\circ} \mathrm{C}$ and postfixed in $1 \%$ osmium tetroxide for one hour. The samples were dehydrated in increasing concentrations of ethanol (from 50\%, 70\%, 90\% to 100\%) and were criticalpoint-dried. Cover slips were mounted on aluminium stubs before being sputter coated with a thin layer of gold in a SC500, Bio-Rad sputter coater (Bio-Rad, BioRad Laboratories Pty Ltd, New south wales, Australia) before examination using a FEI Quanta 200 scanning electron microscope (FEI, Hillsboro, OR, USA). Backscatter imaging on in vivo samples was performed as described previously [6].

\section{Immunoflouresence}

To examine focal adhesion formation and cytoskeletal organisation, osteocytes cultured on normal and OA ECM substrates were fixed in $4 \%$ paraformaldehyde
(pH 7.4 in PBS) for 10 minutes, and then permeabilised with $0.2 \%$ Triton $\mathrm{X}-100$ for 10 minutes. After washing and blocking with 1\% BSA, cells were incubated with primary antibody vinculin $5 \mu \mathrm{g} / \mathrm{ml}$ (Sigma Aldrich, New south wales, Australia), SOST $5 \mu \mathrm{g} / \mathrm{ml}$ (R\&D Systems, Sapphire Biosciences Pty Ltd, New south wales, Australia), DMP1 $5 \mu \mathrm{g} / \mathrm{ml}$ (gift from Professor Jian Feng) and E11 $5 \mu \mathrm{g} / \mathrm{ml}$ at $4^{\circ} \mathrm{C}$ overnight followed by secondary antibody (Alexa Fluor 488-labeled goat anti mouse immunoglobulin G (IgG), Invitrogen) incubation. F-actin distribution was visualised using Alexa Fluor 568 nmlabeled phalloidin (Invitrogen) staining. The samples were washed with 1X PBS three times, blown dry with air and mounted with Prolong Gold antifade solution containing DAPI for cellular nuclei staining and fluorescence preservation. Samples were then visualised using a DeltaVision PDV microscopy system equipped with an Olympus IX70 inverted microscope (Olympus). After acquisition of the $Z$-series images, they were deconvolved using the software attached in the system (Softworx; Applied Precision). The shapes of the fluorescent signals in osteocytes cultured on the normal and OA matrix were analysed using the integrated morphometry shape factor analysis of Metamorph software version 7.1.2 (Universal Imaging). This algorithm assigns a value from 0 to 1 , describing the shape of the fluorescence signal where a perfect circle attains a value of 1 and a line is assigned a 0 .

\section{Cell attachment assays}

MLOY4 cells were seeded directly on normal and OA matrices with a seeding density of 10,000 viable cells per well. Cells were allowed to attach in the humidified incubator for two different time periods, 3 and 24 hours. At the end of each culture period cells were stained for 10 minutes with $0.2 \%$ crystal violet (Sigma) in $20 \%$ methanol. After washing with $\mathrm{H}_{2} \mathrm{O}$, plates were dried overnight at room temperature. Cells were dissolved in 1\% SDS $(150 \mu \mathrm{l})$, and the absorbance at $570 \mathrm{~nm}$ was measured.

\section{Cell proliferation assays}

Cell proliferation was determined using the CyQUANT NF Cell Proliferation Assay Kit (Molecular Probes, Invitrogen) at 48 hours of MLOY4 cell culture on normal and OA matrices according to the manufacturer's instructions. Plates were then analysed by using a microplate reader (excitation: $485 \mathrm{~nm}$, emission: $520 \mathrm{~nm}$ ).

\section{Apoptosis assays}

The viability of MLOY4 cells was quantified by flow cytometry with the AnnexinV-FLOUOS apoptosis staining kit (Roche Applied Science, Germany, Roche Australia PVT LTD, New south wales, Australia) following the manufacturer's protocol. 


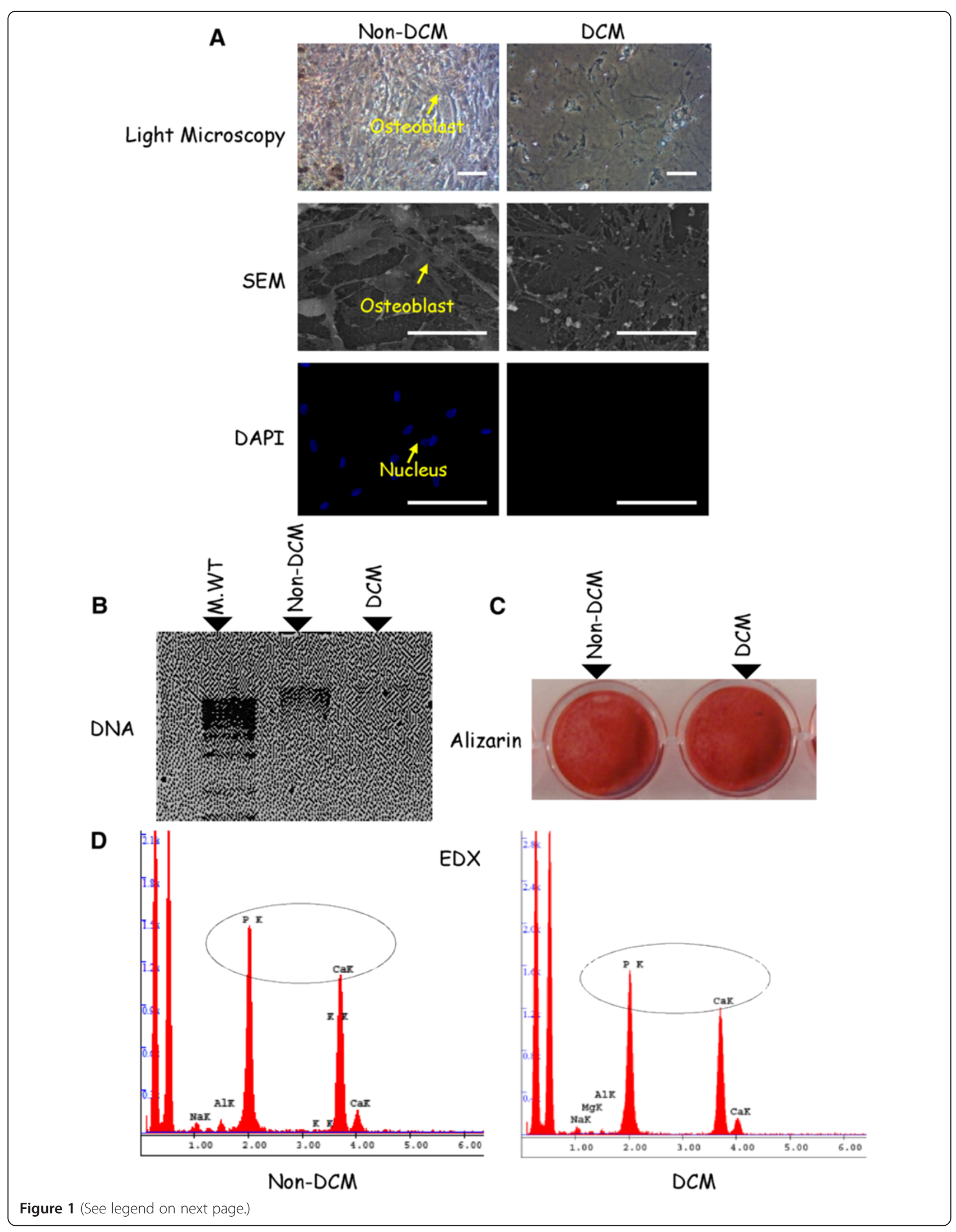


(See figure on previous page.)

Figure 1 Confirmation of de-cellularisation in osteoblast secreted matrices. (A) Light microscopy (scale bar $=100 \mu \mathrm{m}$ ) and SEM techniques were performed following the de-celluarisation of normal matrices. Non de-celluarised osteoblasts are shown as controls. (scale bar: 20 Mm). DAPI staining of de-cellularised matrices show no nuclear remnants and non de-cellularised matrices show intact nuclei (scale bar: $50 \mu m$ ). (B) DNA analysis of the normal osteoblast derived matrices confirmed the removal of nucleic acids in the lysates of de-cellularised matrices. (C, D) Alizarin red staining and the EDX technique were performed to see the mineral content of the normal and OA de-cellularised matrices. All results (from A to D) are representative of matrices produced from four different patients each time with two replicates. Non DCM: Non de-cellularised matrix; DCM: de-cellularised matrix. DAPI, 4',6-diamidino-2-phenylindole; EDX, energy dispersion X-ray; OA, osteoarthritis; SEM, scanning electron microssopy.

\section{Quantitative real time PCR (qRT-PCR), zymography, and western blotting}

qPCR, zymography and western blotting techniques were performed as described in our previously published studies [12-14,20].

\section{Statistical analysis}

Comparisons between groups were carried out by Student's $t$-test and for multiple comparisons analysis of variance (ANOVA) was used where $P \leq 0.05$ was considered significant. Results are presented as a mean \pm standard deviation (SD).

\section{Results}

\section{Confirmation of de-celluarisation}

Light microscopy and SEM analysis of de-cellularised normal matrices confirmed the intact architecture and absence of cells following the processing. Nuclear remnants could not be detected by DAPI staining, indicating satisfactory de-cellularisation of osteoblasts leaving the intact matrices. Non de-cellularised matrices were observed in parallel, showing the cell nuclear staining (Figure 1A). The absence of DNA in the matrix lysates further confirmed that approximately $99 \%$ of the DNA was removed by the de-cellularisation process (Figure 1B). Alizarin red staining confirmed the presence of mineralisation nodules after de-cellularisation (Figure 1C). Furthermore, EDX showed clear peaks for both phosphorus and calcium confirming the presence of calcium phosphate in the de-cellularised matrices obtained from the osteogenic culture of SBOs (Figure 1D). Hence, based on these results, we determined that the de-cellularisation protocol produced an acellular matrix scaffold that retains the gross, microstructural, and ultrastructural properties of the native bone tissue ECM microenvironment.

\section{Characterisation of ECM secreted by normal and OA osteoblasts}

First, at the gene level, bone regulating genes such as runt-related transcription factor (RUNX2), osteocalcin $(O C N)$, alkaline phosphatase $(A L P)$ and bone sialoprotein $(B S P)$ were up-regulated in OA osteoblasts compared with normal osteoblasts (Figure 2A). Furthermore, matrix mineralisation of normal osteoblasts was higher compared to
OA osteoblasts (Figure 2B). Immunoblotting was performed on the normal and OA derived de-cellularised matrices to address changes in ECM constitution specifically focussing on bone matrix RGD glycoproteins (which specifically recognise integrins). The expression of fibronectin (FN), versican and laminin (LN) was significantly higher in normal osteoblast derived matrices compared to OA osteoblast derived matrices. Surprisingly, the largest amounts of osteopontin (OPN) were present in OA osteoblast derived matrices compared to normal. COLIII was detectable in both normal and OA matrices; however this molecule did not show a significant difference (Figure $2 \mathrm{C}$ to $\mathrm{G})$. These results demonstrate that the ECM derived from normal and OA osteoblasts were different with respect to its composition. After five weeks of osteogenic differentiation, SEM and type I collagen (COL1) immunostaining analysis demonstrate that the de-cellularised matrix formed by the OA osteoblasts had a less preserved matrix organisation and alignment compared to normal matrix. In accordance with the results obtained from the in vitro studies, matrix mineral distribution was disorganised, showing a woven appearance in tibial severe OA subchondral bone derived from patients undergoing knee replacement surgery (Figure $2 \mathrm{H}$ ).

\section{Differential cell-matrix adhesions regulate morphology of osteocytic cells scattered within the normal and osteoarthritis osteoblast derived extracellular matrix}

The morphology of osteocytic cells cultured on normal and OA osteoblast derived ECMs was examined via light microscopy, SEM analysis and confocal microscopy. First, basic microscopic observations of osteocytic cell populations revealed striking morphological differences when grown on normal and OA matrices. Osteocytic cells that were cultured on OA matrix typically displayed a round morphology, tended to have less protrusive extensions and their cytoplasm was wider. On the other hand, osteocytic cells that were gown on normal matrix exhibited a narrow and elongated dendritic morphology. Similarly, Alizarin red combined with haematoxylin staining revealed well organised osteocytic cells in the normal bone matrix compared to OA (Figure 3A). Based on these preliminary differences, SEM was used to further 


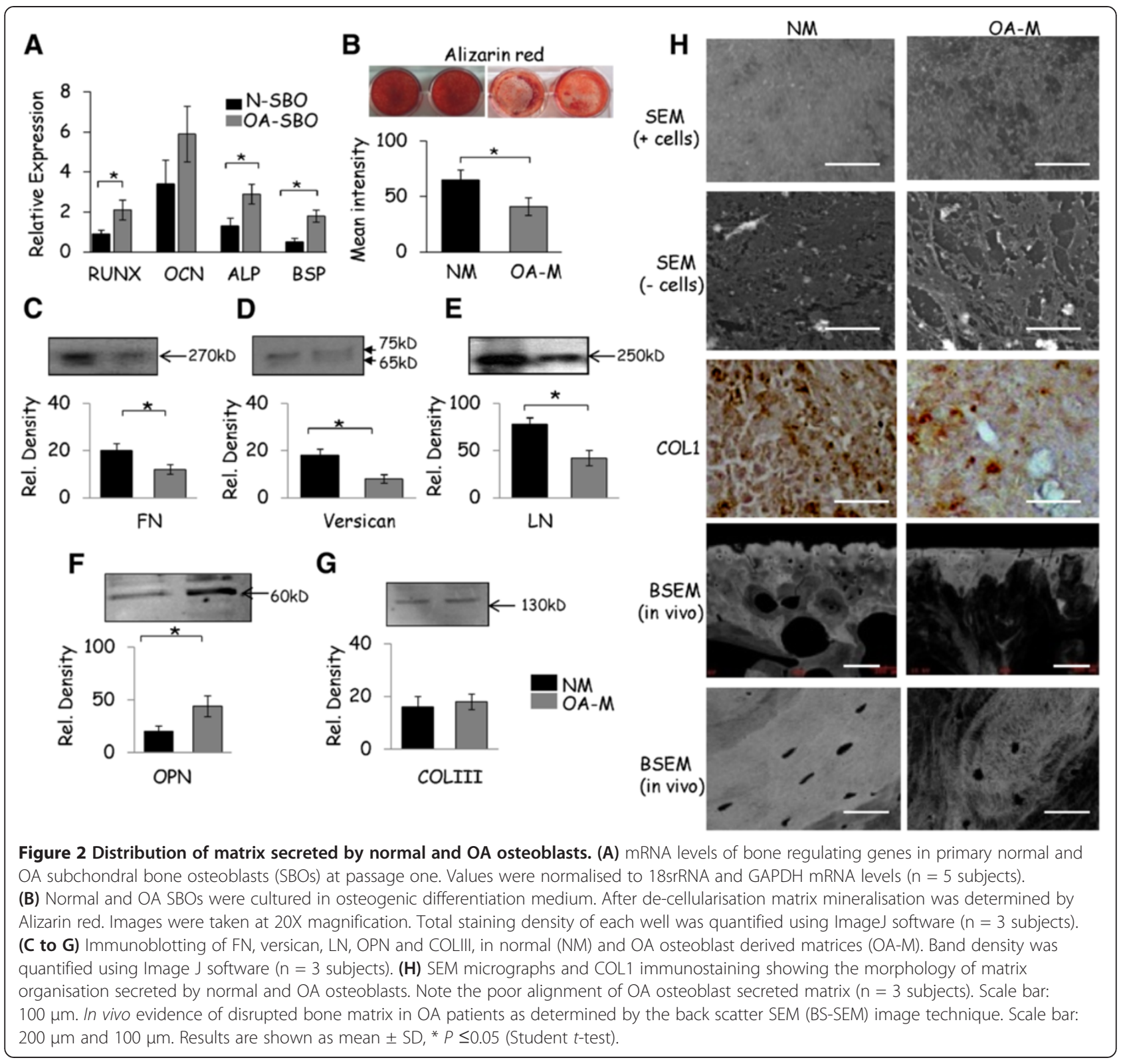

confirm these changes. SEM microphotographs showed that the osteocytic cells grown on the normal matrix revealed a dense network of dendrites, adhesion to the matrix surface and interaction between cells, whereas the cells that were grown on the OA matrices revealed round, rough and lysed morphology with poor dendrite formation and matrix alignment. We next examined the in vivo morphology of osteocytes in the OA subchondral bone derived from knee replacement surgeries. SEM revealed that the osteocytes in OA samples were markedly deformed with a rough, lysed and rounded appearance with very few dendrites compared to spindle shaped, well organised and well connected osteocytes in the relatively normal specimens, closely resembling the results obtained from the in vitro cultures as shown above. These results suggest that we could closely mimic the in vivo osteocyte and matrix interactions using the de-cellularised matrices (Figure 3B).

These differences in morphology suggest underlying differences in the organisation of the respective cytoskeletons. Osteocytic cells that were grown on normal matrix had straight actin fibres, which were visualised by phalloidin. When the cells were grown on the OA matrix, actin filament fibres were observed randomly and visually no fibres were present at the center of the osteocytic cells (Figure 3C).

The formation of focal adhesion complexes is a prerequisite for cell-ECM adhesion. Therefore, it is possible that the altered cell morphology capabilities of osteocytic cells on the OA matrix may result from 


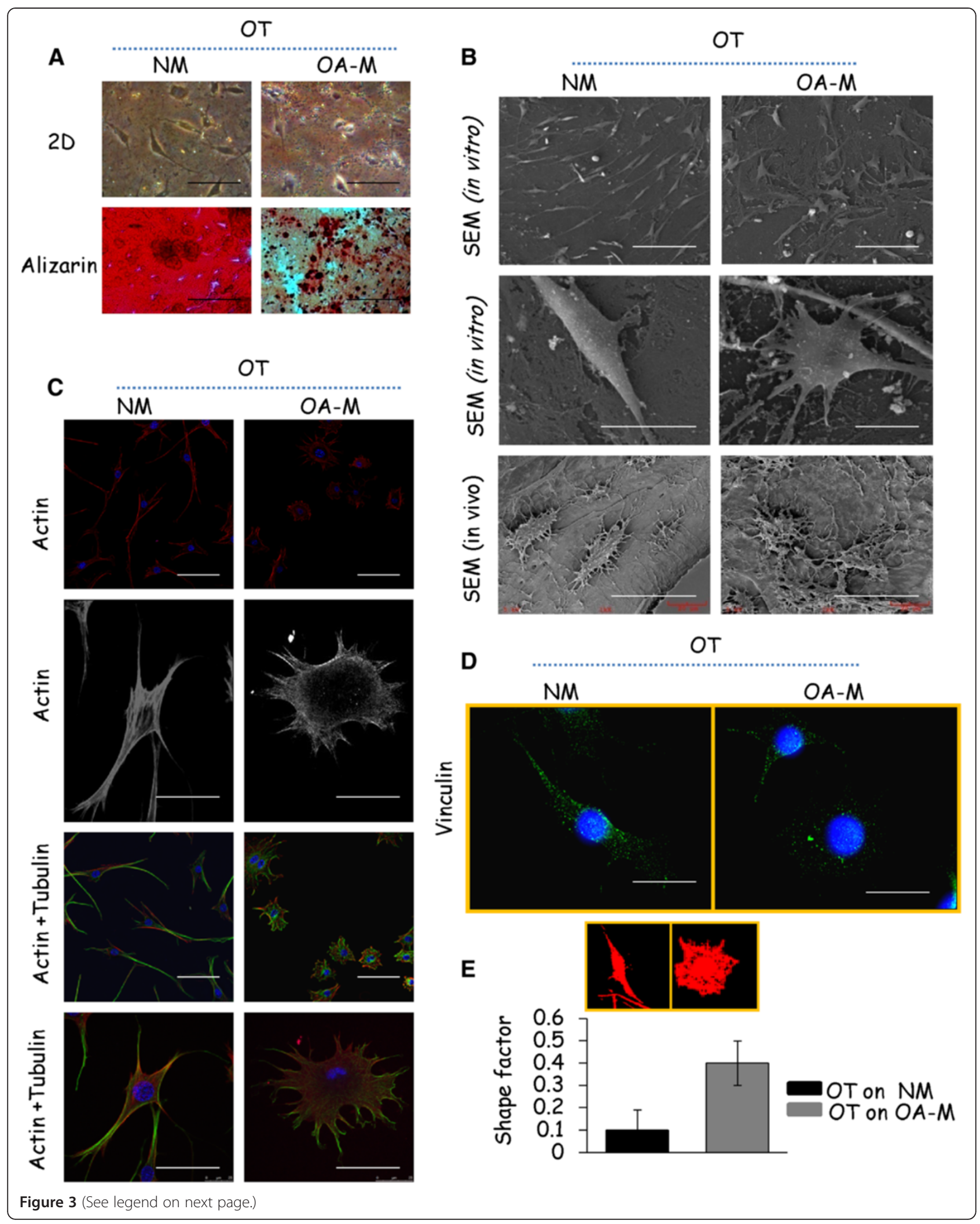


(See figure on previous page.)

Figure 3 Morphological changes of osteocytic cells in response to normal and OA osteoblast-derived matrices. (A) Cells cultured in 2D showed rounded osteocyte morphology when grown on the OA matrices. Alizarin red combined with the haematoxylin staining showed a well aligned osteocytic cell distribution within normal matrix (NM) compared to OA matrix (OA-M). Magnification: 20X. (B) SEM (Scale bar: 100 Hm for higher and $20 \mu \mathrm{m}$ for lower magnification) and (C) confocal images (Scale bar: $50 \mu \mathrm{m}$ for higher and $25 \mu \mathrm{m}$ for lower magnification) indicated diversified morphologies of osteocytic cells grown on OA osteoblast derived matrices. Actin filaments are shown in red, microtubules in green and the nuclei in blue. (D) Representative micrographs of vinculin immunofluorescence (green) staining in osteocytic cells grown on normal and OA matrices. Scale bars: $25 \mu \mathrm{m}$. (E) Shape factor analysis of the confocal images (phalloidin only) was performed with the Metamorph software. At least 15 cells from three different experiments were analyzed. All images and graphs (A-E) are representative of experiments performed on osteocytic cells that were grown on four different normal and OA patient-derived matrices. Results are shown as mean \pm SD. Student's $t$ test, $\mathrm{P}<0.05$.

alterations in focal adhesion functions. We thus used immunofluorescence staining to examine the expression of focal adhesions. As shown in Figure 3D, osteocytic cells grown on normal matrices formed multiple focal adhesion structures at plasma membranes and cytoplasm, as visualised by staining for vinculin, an abundant cytoskeleton protein localised at focal adhesions. In marked contrast, osteocytic cells on an OA matrix showed fewer focal adhesion contact points. Confocal images of osteocytic cells shown in Figure $3 \mathrm{C}$ were quantitatively analysed for shape factor. Cell shape factor changed from 0.1 to 0.4, indicating a transformation from a non-circular spreading pattern as the cells developed spindle shaped morphology to a rounded/circle pattern when grown on an OA matrix (Figure 3E).

\section{Altered functional and gene expression characteristics of osteocytic cells grown on osteoarthritis osteoblast- derived matrices}

To understand the regulation of osteocyte function on the normal and OA matrices, we compared osteocyte specific gene expression, cell attachment, apoptosis and Matrix metalloproteinases (MMP-2) and -9 production. Using real-time PCR, we analysed a panel of characteristic osteocyte markers after 48 hours of osteocytic cell culture on normal and OA matrices. We observed that the expression of matrix extracellular phosphoglycoprotein (MEPE) and E11 were upregulated significantly when osteocytic cells were grown on OA matrices. On the other hand, the expression of phosphate-regulating gene with homology to endopeptidases on $\mathrm{X}$ chromosome (PHEX) remained at similar levels with no differences between normal and OA matrices. In contrast, DMP1 and SOST were significantly downregulated in osteocytic cells that were cultured on the OA matrices compared to those that were grown on normal matrix. (Figure 4A to E). Of note, although the MLOY4 cell line is a very powerful tool for the study of osteocytes in vitro [21-23], there are known differences between primary osteocytes and the immortalised MLOY4 cell line. For example, MLOY4 cells express low levels of DMP1 and SOST, while osteocytes are known to express these genes in vivo $[24,25]$. In this study, we observed that MLOY4 cells cultured on the collagen I coated flasks expressed relatively low levels of dentin matrix protein 1 (DMP1) and sclerostin (SOST) (data not shown); however, when these cells were grown on the normal and OA matrices we found a significant increase in the expression of these proteins compared to those grown on the COL1 coated flasks. These results suggest that the embedding of MLOY4 cells in the mineralised matrices may significantly enhance the expression of these mature osteocyte markers, closely mimicking the native bone matrix osteocyte phenotype. Confocal microscopy imaging of the DMP1, SOST and E11 demonstrated trends similar to those seen at the gene expression (Figure 4F, G,H) level. When the ability of cell attachment was compared between normal and OA matrices, at three hours osteocytic cells on normal and OA matrices had settled onto their respective substrates and had a rounded morphology (data not shown). However, at 24 hours the osteocytic cells were attached more on normal matrix compared to those that were grown on the OA matrix as determined by the crystal violet adhesion assay (Figure 4I). Because we observed a lysed morphology of osteocytic cells on the OA matrix, we compared the expression of MMP-2 and MMP-9 by zymography as these molecules are well known to be involved in cell-matrix adhesions. We found higher levels of MMP-2 and MMP-9 in cells that were cultured on the OA matrices (Figure 4J). Furthermore, osteocytic cells exposed to OA bone matrix appeared to be more sensitive to apoptosis than those cultured on the normal matrices (Figure 4K).

\section{Expression of integrin $\beta 1-F A K$ signalling in osteocytic cells cultured in normal and osteoarthritis bone matrices}

In immunohistochemistry analysis, in vivo osteocytes in relatively normal human subchondral bone expressed higher levels of integrin $\beta 1$ compared to osteocytes from OA patients, indicating the altered expression of this molecule in OA osteocytes. This downregulation of integrin $\beta 1$ protein expression in human OA bone tissue lysate was further confirmed by immunoblotting (Figure 5A,B). Furthermore, the expression of integrin $\beta 1$ was significantly higher in osteocytic cells that were grown on the normal matrices compared to OA matrices (OA-M). On the other hand, the expression levels of $\alpha \mathrm{V} \beta 5$ and $\alpha \mathrm{V} \beta 3$ integrins 


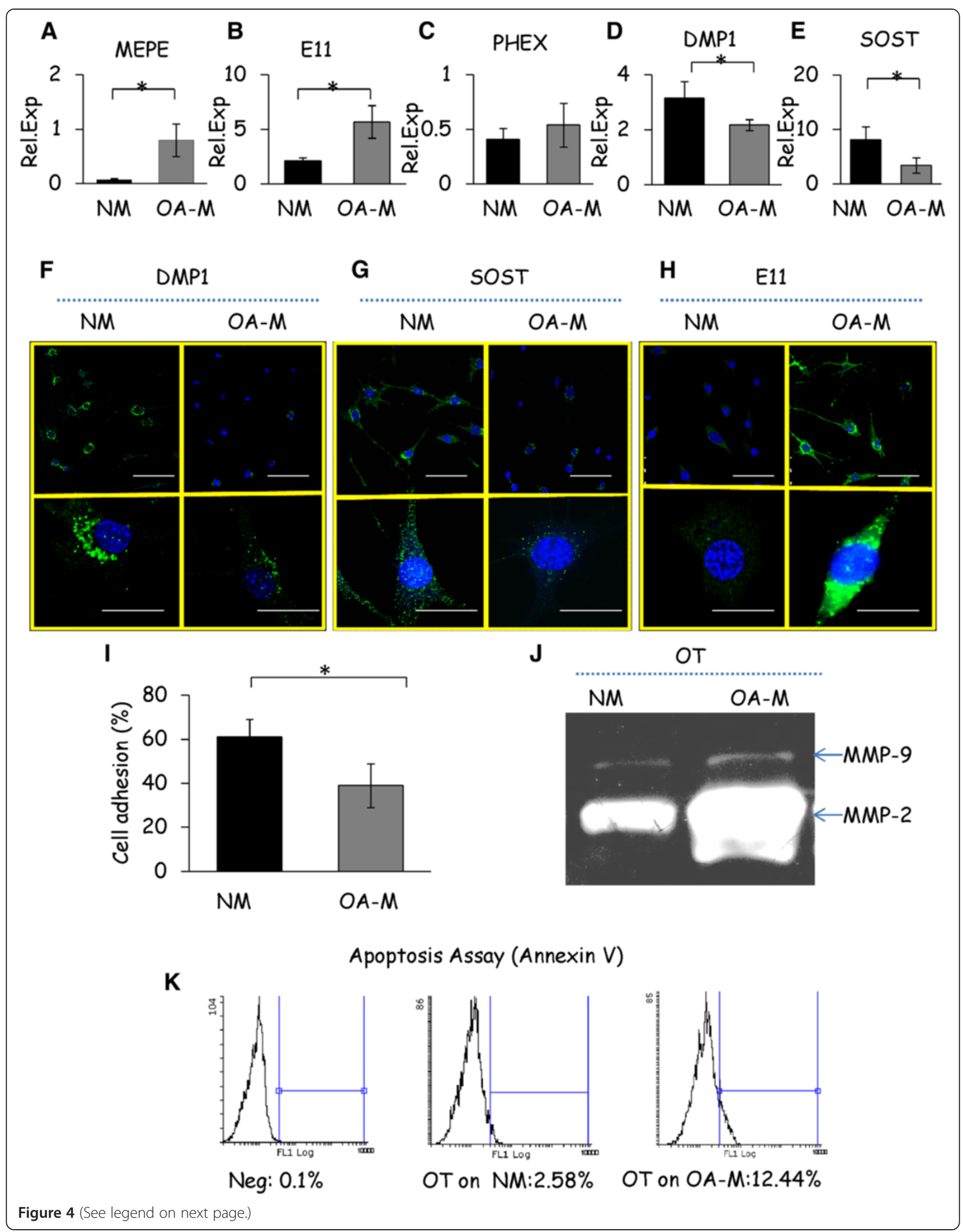


(See figure on previous page.)

Figure 4 Functional and gene associated changes of osteocytic cells (OT) in response to normal (NM) and OA osteoblast derived matrices (OA-M). (A to E) Gene expression profiles of osteocyte markers were determined by qRT-PCR in osteocytic cells cultured either on normal or OA matrices for 48 hours. The results are expressed as relative gene expression after normalization using the 18srRNA and GAPDH housekeeping gene. (F to $\mathbf{H})$ Protein localisation of DMP1, SOST and E11 osteocyte markers was determined by confocal microscopy (Scale bar: $50 \mu \mathrm{m}$ for higher and $25 \mu \mathrm{m}$ for lower magnification). (I) Cell adhesion of osteocytic cells cultured on normal and OA matrices was determined by staining with crystal violet. (J) Zymographic analysis shows the expression of MMP2 and MMP9 in osteocyte conditioned media collected after 24 hours of incubation after culturing on normal and OA matrices. (K) The flow cytometric apoptosis analysis of osteocytic cells on normal and OA matrices was determined by Annexin V/PI staining. All images and graphs are representative of experiments performed on osteocytic cells cultured on matrices derived from four different normal and OA patients. Results are shown as mean \pm SD, ${ }^{*} P \leq 0.05$ by Student's $t$-test.

that also recognise RGD remained unchanged (Figure 5C). Integrin $\beta 1$ can serve as an important regulator of downstream signalling. We, therefore, examined modifications of the intracellular signalling cascade in osteocytic cells after exposure to normal and OA matrices. The experiments concentrated on FAK signalling pathways which are known to be involved in cell-matrix regulation. Also, compared to the OA matrices the osteocytic cells cultured on normal matrices evoked upregulation of FAK phosphorylation (Figure 5D). Furthermore, integrin $\beta 1$ inhibition using blocking antibodies led to FAK dephosphorylation even when osteocytic cells were cultured on normal matrices indicating that the phosphorylation of FAK was dependent on integrin $\beta 1$ activation (Figure $5 \mathrm{E}$ ).

\section{Integrin $\beta 1$ blocking diminished phenotypic and genotypic capacity of osteocytic cells attached on the normal matrices}

Next we examined the relevance of integrin $\beta 1$ in mediating the phenotypic and genotypic responses of osteocytic cells cultured on the normal matrices by using blocking antibodies. In this context, as determined by SEM and confocal microscopy, the morphology of osteocytic cells grown on normal matrices in the presence of integrin $\beta 1$ blocking antibodies was drastically changed to a phenotype similar to cells that were grown on the OA matrices, demonstrating a link between integrin $\beta 1$ and altered osteocyte cell-matrix interaction (Figure 6A,B). Furthermore, osteocytic cell adhesion was decreased in the

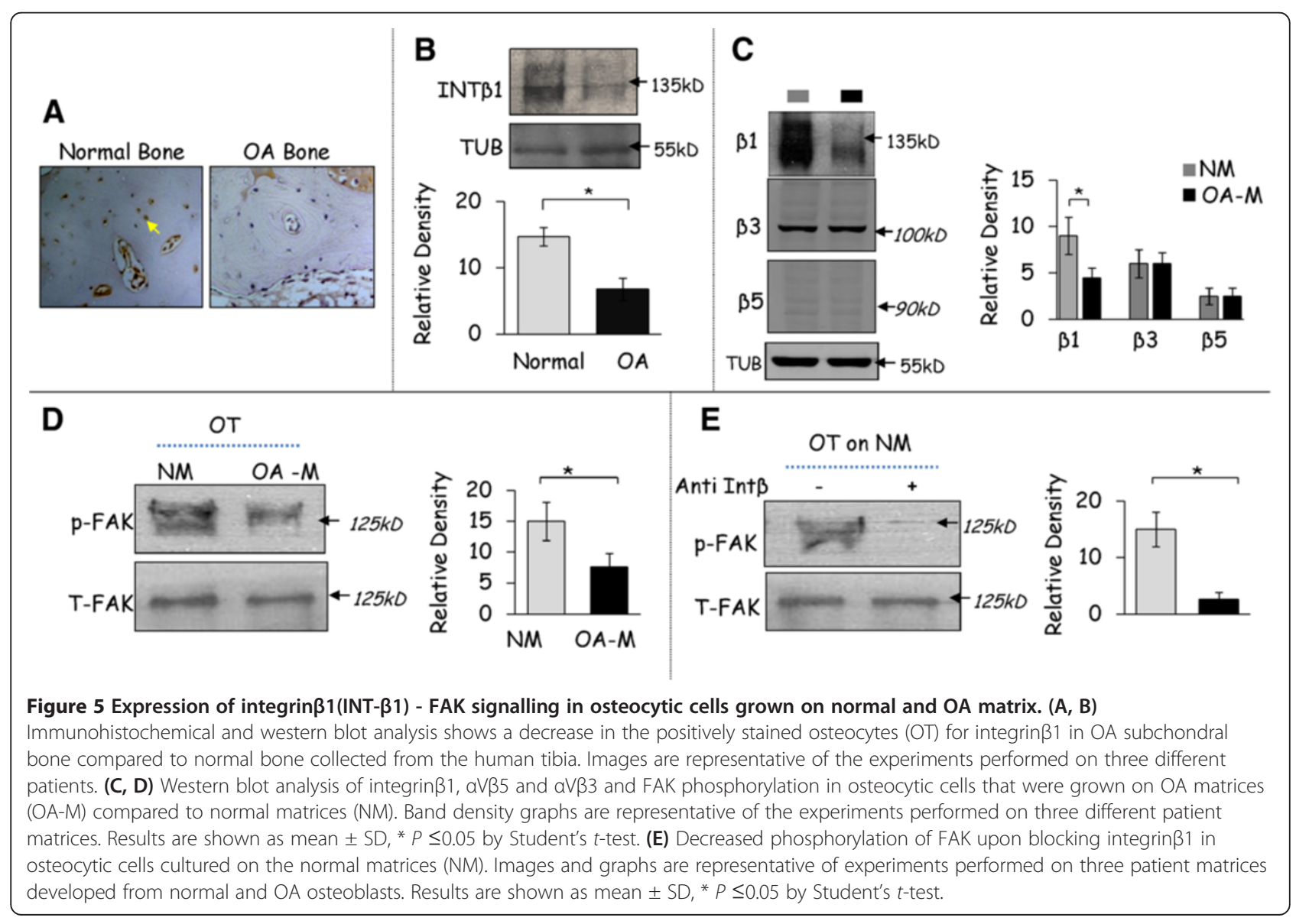




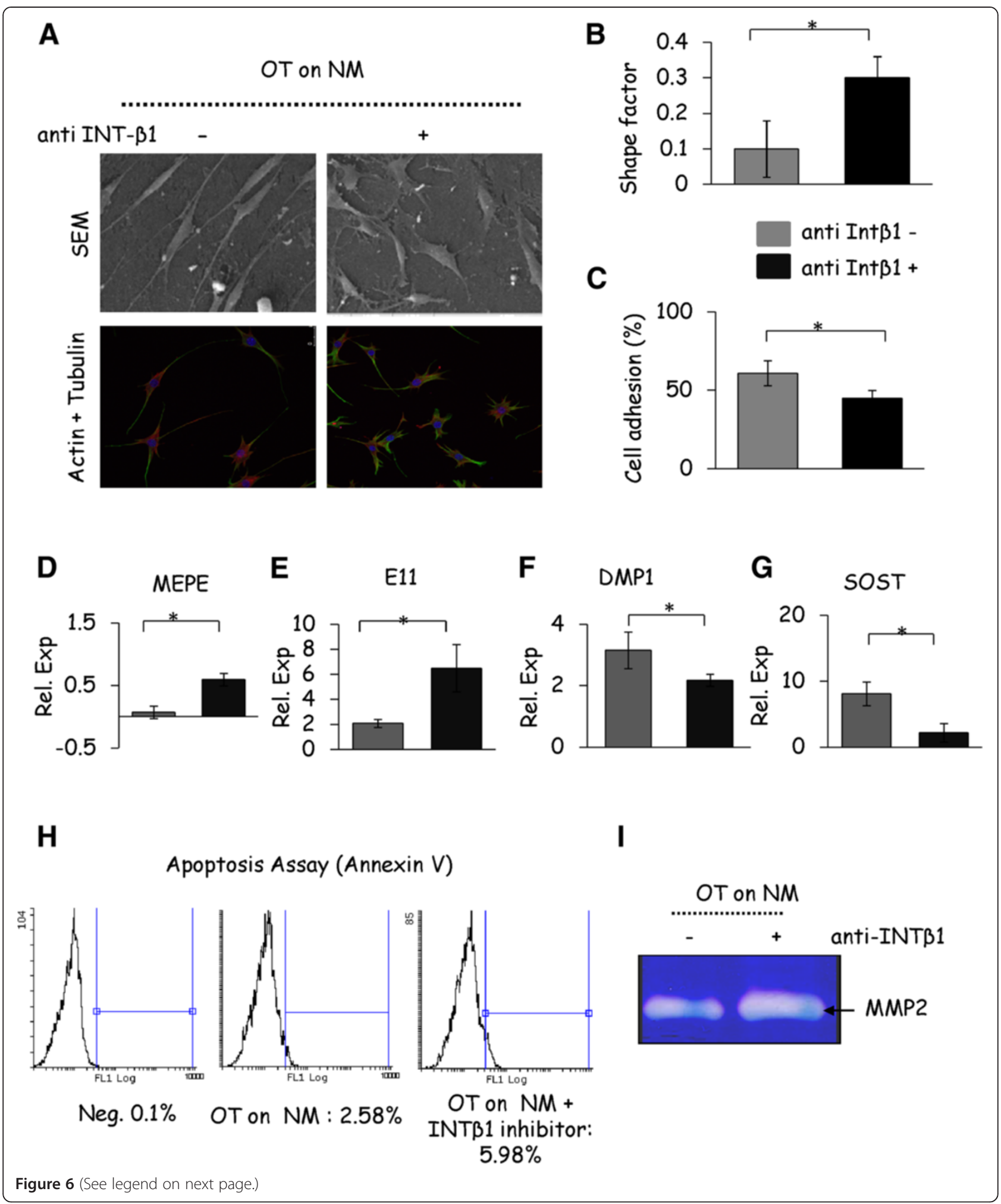

presence of integrin $\beta 1$ blocking antibodies (Figure 6C). In addition, the osteocyte markers E11 and MEPE were upregulated and genes, such as DMP1 and SOST, were significantly downregulated upon blocking of integrin $\beta 1$
(Figure 6D to G). Likewise, blocking antibodies against integrin $\beta 1$ increased osteocyte apoptosis (Figure $6 \mathrm{H}$ ) and MMP production (Figure 6I). These results together suggest an important role of integrin $\beta 1$ in mediating the 
(See figure on previous page.)

Figure 6 Integrin $\beta 1$ (INT- $\beta 1$ ) blocking disables the morphology and function of osteocytic cells cultured on normal matrices.

(A) SEM and confocal images illustrating distinct morphological changes induced by integrin $\beta 1$ blocking in osteocytic cells cultured on the normal matrices (NM). (Scale bars: $50 \mu \mathrm{m}$ ). (B) Shape factor determined on the osteocytic cells cultured on normal matrices upon integrin $\beta 1$ blocking. (C) Cell adhesion determined on the osteocytic cells grown on the normal matrices upon integrin $\beta 1$ blocking (D to G) Gene expression profiles of osteocyte markers as determined by qRT-PCR in the presence of integrin $\beta 1$ blocking antibodies on normal matrix. The results are expressed as relative gene expression after normalisation using the 18srRNA and GAPDH housekeeping gene. (H) Osteocytic cells grown on the normal matrices were treated with integrin $\beta 1$ blocking antibody and incubated for 24 hours, and then detection of apoptosis was performed by flow cytometry. (I) Zymographic analysis shows the expression of MMP2 in osteocytic cell conditioned media collected after 24 hours of incubation after culturing on normal matrices in the presence or absence of integrin $\beta 1$ blocking antibody. All images and graphs are repremsentative of experiments performed on osteocytic cells cultured on matrices derived from three different normal patients. Results are shown as mean $\pm \mathrm{SD},{ }^{*} P \leq 0.05$ by Student's $t$-test.

osteocyte and cell matrix interactions under normal conditions.

\section{Discussion}

It is now well accepted that joint cartilage degeneration is associated with intensified remodelling of the subchondral bone and increased bone stiffness [26]. Understanding which cells/molecules are involved in bone sclerosis could help us find ways to manipulate such molecules to slow down the progression of OA.

Earlier studies have shown that the de-cellularised matrix produced by osteoblasts is able to induce attachment and spreading of many adherent cell types, including mesenchymal stem cells [27-29]. In principle, the use of $\mathrm{NH} 4 \mathrm{OH}$ leads to hypoxia and subsequent cell lysis leaving the matrix intact. It is thus regarded as an in vitro model to study the molecular basis of cell attachment, spreading and signalling from the ECM. In the present study, we confirmed that de-cellularised osteoblast matrices represent the in vivo bone matrix characterised by accumulation of the mineral content and expression of various collagenous and non-collagenous proteins, thus providing the ideal environment to study cell-matrix interactions. First, we observed significant differences in the mineral content of matrices secreted by normal and OA osteoblasts. These results are in agreement with previous reports suggesting the abnormally low mineralisation of OA osteoblasts [30]. The present study also showed that human osteoblast ECM derived from primary bone cell cultures has the capacity to produce a wide range of matrix proteins. Surprisingly, we observed that the levels of fibronectin, laminin and versican were decreased in OA matrices compared to normal. In general, fibronectin is presumed to contribute more to general structure and load bearing [31], whereas versican and laminin interact with other matrix components as well as with cell surface adhesion receptors via well-defined domains [32]. Low expression of these molecules is suggestive of abnormal ECM composition in OA matrices. Previous studies demonstrated an increase of COL1 in OA osteoblasts at the cellular level [33]. In this study, the matrix deposited by OA osteoblasts showed a dispersed COL1 staining compared to normal matrix. These results corroborate the results of studies showing disorganised collagen fibres in OA bone compared to normal [34]. Furthermore, although not statistically significant, we observed that expression of $\mathrm{OCN}$ was higher in OA patients. However, many other studies showed a significant increase of OCN in OA patients $[30,35]$. This disparity in the results could be attributed to biological variation, process variation and system variation.

Based on the above results, it is apparent that the ECM secreted by normal and OA osteoblasts is different. We next tested whether these matrix differences can contribute to the poor morphological and phenotypic properties of osteocytes seen in OA bone. Our results suggest that osteocyte cell lines, such as MLOY4, could be altered in response to the ECM of OA osteoblasts compared to the ECM generated from normal osteoblasts. At the gene expression level, when osteocytic cells were grown on OA matrices a decrease in DMP1 and SOST expression and an increase in E11 and MEPE expression were found. Although E11, MEPE, DMP1 and SOST are known markers of the osteocyte, each gene has a distinct function and their expression levels change according to the specific stage of differentiation. Osteocyte expression of SOST [36] and DMP [37] is a delayed event and it is produced only by osteocytes after they become embedded deeply in matrix that has been fully mineralised. Our results showed that OA osteoblasts failed to lay a proper mineral or matrix. Therefore, these matrix properties might have influenced the behaviour of osteocytes, stopping them from expressing those mature markers; whereas E11 and MEPE are early markers and they start to express when osteocytes are in the process of embedding into the matrix that is not fully mineralised [38]. Failure of osteocytic cells to produce these proteins when cultured on the OA matrix also suggests a state of immature osteocytes. These results together suggest that in OA matrices osteocytic cells remained in an immature phenotype; however, when they were cultured on the normal matrices, the osteocytes differentiated from an immature to mature phenotype. Likewise, on the OA matrices an increase in 
osteocytic cell death was observed which can possibly be related to increased skeletal fragility, linked to the loss of ability to sense microdamage and/or signal repair of in vivo bone [39].

The observation that the morphological and phenotypic characteristics of osteocytic cells attached to the normal and the OA matrices differ from each other, suggests that specific signalling pathways must arise or alter between matrix and cells. Of note, we observed that the expression of integrin $\beta 1$ was much less in osteocytic cells that were cultured on the OA matrices compared to normal matrices indicating that the decrease of integrin $\beta 1$ expression could be responsible for the observed phenotypic changes of osteocytes in OA.

How and to what extent integrin $\beta 1$ is involved in the transduction of a matrix signal to modulate osteocyte function was investigated by a series of further experiments. We found that the blocking integrin $\beta 1$ activity promoted the elongated cell morphology to a rounded phenotype even when osteocytic cells are cultured on normal osteoblast matrices. This series of events occurred via the down regulation of FAK phosphorylation levels. The effect of integrin $\beta 1$ blocking on cell morphology was possibly due to FAK signals leading to actin contractility events and the dynamic regulation of viniculin. Normally, integrin $\beta 1$-FAK signalling, apart from playing a role as a hook to attach the cell to matrix, generates a signal to enforce the cell towards functions such as attachment, apoptosis and differentiation. In this study, we observed that osteocytic cells on OA matrices and integrin $\beta 1$ blocking showed an increased apoptosis and altered osteocyte gene expression, indicating that the expression of integrin $\beta 1$-FAK signalling is important to maintain the normal osteocyte-matrix interactions. In vitro data presented here are consistent with results from an integrin $\beta 1$ negative mouse model in which mice exhibited less mineralised bone, reduced tibial curvature and decreased femoral strength [40]. Thus, this study provides evidence that integrin $\beta 1$ may initiate intracellular signals either by organisation of the cytoskeleton and alteration of cell shape or through mechanisms akin to osteocyte signalling. In this study, we observed that the expression of $\mathrm{V} \beta 3$ and $\mathrm{V} \beta 5$ were not changed. Although different integrin receptors perform common functions and can share identical ligands, each member seems to be highly specific, since mice carrying gene deletions of the different integrin chains often show non-overlapping phenotypes [41]. We are confident that there might be other integrins participating in the interaction and further studies are warranted to determine their role.

\section{Conclusion}

In summary, this study demonstrated that ECM from OA osteoblasts can induce significant alteration of osteocytic cells via a focal adhesion mediated integrin $\beta 1$-FAK cell signalling pathway, a possible mechanism of OA subchondral bone sclerosis and OA progression.

\section{Abbreviations}

ALP: Alkaline phosphatase; BSA: Bovine serum albumin; BSEM: Back scattered electron microscopy; BSP: Bone sialo protein; COL1: Type I collagen; COLIII: Type III collagen; DAPI: 4',6-diamidino-2-phenylindole; DCM: Decellularised matrix; DMP: Dentin matrix protein; DMP1: Dentin matrix protein 1; (D)MEM: (Dulbecco's)_modified Eagle's medium; ECM: Extracellular matrix; EDX: Energy dispersive X-ray; FAK: Focal adhesion kinase; FN: Fibronectin; INTb1: Integrin beta1; LN: Laminin; MEPE: Matrix extracellular phosphoglycoprotein; MMP: Matrix metalloproteinases; OA: Osteoarthritis matrix; OCN: osteocalcin; OPN: Osteopontin; OT: Osteocytes; PBS: Phosphatebuffered saline; PHEX: Phosphate-regulating neutral endopeptidase; qPCR: Quantitative polymerase chain reaction; SBO: Subchondral bone osteoblasts; SEM: Scanning electron microscopy; SOST: Sclerostin; TUB: Tubulin.

\section{Competing interests}

The authors declare that they have no competing interests.

\section{Authors' contributions}

IP planned the studies, performed experiments, analysed data and wrote the manuscript. SF, WG, SP, JF and RC did experiments and analysed data. YX supervised the project, planned studies, analysed data and wrote the manuscript. All authors have been involved in drafting the manuscript or in revising it critically for important intellectual content and have read and approved the final manuscript.

\section{Acknowledgement}

This study is supported by the Prince Charles Hospital Foundation (MS2010-02), NHMRC project fund (APP1032738), and the Australian Orthopaedics Association Research Foundation. The authors would like to thank, Dr Anna Taubenberger for her kind inputs in the de-cellularisation protocols and Dr Abhishek Kashyap for provision of integrin antibodies.

\section{Author details}

'Institute of Health and Biomedical Innovation, Queensland University of Technology, Kelvin Grove Campus, Brisbane, Queensland 4059, Australia. ${ }^{2}$ Prince Charles Hospital, Brisbane, Queensland, Australia. ${ }^{3} T$ exas A\&M Health Science Center, H, Round Rock, TX, USA.

Received: 7 March 2013 Accepted: 17 September 2013

Published: 9 October 2013

\section{References}

1. Green J, Schotland S, Stauber DJ, Kleeman CR, Clemens TL: Cell-matrix interaction in bone: type I collagen modulates signal transduction in osteoblast-like cells. Am J Physiol 1995, 268:C1090-C1103.

2. Popov C, Radic T, Haasters F, Prall WC, Aszodi A, Gullberg D, Schieker M, Docheva D: Integrins alpha2beta1 and alpha11beta1 regulate the survival of mesenchymal stem cells on collagen I. Cell Death Dis 2011, 2:e186.

3. Zohar R: Signals between cells and matrix mediate bone regeneration. In Bone Regeneration. Edited by Tal PH. InTech; 2012. Available from: http://www.intechopen.com/books/bone-regeneration/signals-betweencells-and-matrix-mediate-bone-regeneration.

4. Burr DB: The importance of subchondral bone in the progression of osteoarthritis. J Rheumatol Supp/ 2004, 70:77-80.

5. Neve A, Corrado A, Cantatore FP: Osteocytes: central conductors of bone biology in normal and pathological conditions. Acta Physiol (Oxf) 2012, 204:317-330.

6. Jaiprakash A, Prasadam I, Feng JQ, Liu Y, Crawford R, Xiao Y: Phenotypic characterization of osteoarthritic osteocytes from the sclerotic zones: a possible pathological role in subchondral bone sclerosis. Int J Biol Sci 2012, 8:406-417.

7. Lu XL, Huo B, Chiang V, Guo XE: Osteocytic network is more responsive in calcium signaling than osteoblastic network under fluid flow. J Bone Miner Res 2012, 27:563-574. 
8. Bonewald LF: Osteocyte biology: its implications for osteoporosis. J Musculoskelet Neuronal Interact 2004, 4:101-104.

9. Schaffner $P$, Dard MM: Structure and function of RGD peptides involved in bone biology. Cell Mol Life Sci 2003, 60:119-132

10. Robey PG, Fedarko NS, Hefferan TE, Bianco P, Vetter UK, Grzesik W. Friedenstein A, Van der Pluijm G, Mintz KP, Young MF, et al: Structure and molecular regulation of bone matrix proteins. J Bone Miner Res 1993 8:S483-S487.

11. Legate KR, Wickstrom SA, Fassler R: Genetic and cell biological analysis of integrin outside-in signaling. Genes Dev 2009, 23:397-418.

12. Prasadam I, Crawford R, Xiao Y: Aggravation of ADAMTS and matrix metalloproteinase production and role of ERK $1 / 2$ pathway in the interaction of osteoarthritic subchondral bone osteoblasts and articular cartilage chondrocytes - possible pathogenic role in osteoarthritis. J Rheumatol 2012, 39:621-634.

13. Prasadam I, Friis T, Shi W, van Gennip S, Crawford R, Xiao Y: Osteoarthritic cartilage chondrocytes alter subchondral bone osteoblast differentiation via MAPK signalling pathway involving ERK1/2. Bone 2010, 46:226-235

14. Prasadam I, van Gennip S, Friis T, Shi W, Crawford R, Xiao Y: ERK-1/2 and p38 in the regulation of hypertrophic changes of normal articular cartilage chondrocytes induced by osteoarthritic subchondral osteoblasts. Arthritis Rheum 2010, 62:1349-1360.

15. Altman R, Asch E, Bloch D, Bole G, Borenstein D, Brandt K, Christy W Cooke TD, Greenwald R, Hochberg M, et al: Development of criteria for the classification and reporting of osteoarthritis. Classification of osteoarthritis of the knee. Diagnostic and Therapeutic Criteria Committee of the American Rheumatism Association. Arthritis Rheum 1986, 29:1039-1049.

16. Beresford JN, Gallagher JA, Gowen M, McGuire MKB, Poser JW, Russell RG: Human bone cells in culture: a novel system for the investigation of bone cell metabolism. Clin Sci (Colch) 1983, 64:38-39.

17. Beresford JN, Gallagher JA, Poser JW, Russell RG: Production of osteocalcin by human bone cells in vitro. Effects of $1,25(\mathrm{OH}) 2 \mathrm{D} 3,24,25(\mathrm{OH}) 2 \mathrm{D} 3$, parathyroid hormone, and glucocorticoids. Metab Bone Dis Relat Res 1984, 5:229-234.

18. Kato Y, Windle JJ, Koop BA, Mundy GR, Bonewald LF: Establishment of an osteocyte-like cell line, MLO-Y4. J Bone Miner Res 1997, 12:2014-2023.

19. Mao X, Peng H, Ling J, Friis T, Whittaker AK, Crawford R, Xiao Y: Enhanced human bone marrow stromal cell affinity for modified poly(L-lactide) surfaces by the upregulation of adhesion molecular genes. Biomaterials 2009, 30:6903-6911.

20. Prasadam I, Mao X, Wang Y, Shi W, Crawford R, Xiao Y: Inhibition of p38 pathway leads to OA-like changes in a rat animal model. Rheumatology (Oxford) 2012, 51:813-823.

21. Marathe N, Rangaswami H, Zhuang S, Boss GR, Pilz RB: Pro-survival effects of 17 beta-estradiol on osteocytes are mediated by nitric oxide/cGMP via differential actions of CGMP-dependent protein kinases I and II. J Biol Chem 2012, 287:978-988.

22. Thompson WR, Majid AS, Czymmek KJ, Ruff AL, Garcia J, Duncan RL, Farach-Carson MC: Association of the alpha(2)delta(1) subunit with Ca(v) 3.2 enhances membrane expression and regulates mechanically induced ATP release in MLO-Y4 osteocytes. J Bone Miner Res 2011, 26:2125-2139.

23. Burra S, Nicolella DP, Francis WL, Freitas CJ, Mueschke NJ, Poole K, Jiang JX: Dendritic processes of osteocytes are mechanotransducers that induce the opening of hemichannels. Proc Natl Acad Sci U S A 2010 107:13648-13653.

24. Stern AR, Stern MM, Van Dyke ME, Jahn K, Prideaux M, Bonewald LF: Isolation and culture of primary osteocytes from the long bones of skeletally mature and aged mice. Biotechniques 2012, 52:361-373.

25. Paic F, Igwe JC, Nori R, Kronenberg MS, Franceschetti T, Harrington P, Kuo L, Shin DG, Rowe DW, Harris SE, Kalajzic I: Identification of differentially expressed genes between osteoblasts and osteocytes. Bone 2009, 45:682-692.

26. Lajeunesse D, Reboul P: Subchondral bone in osteoarthritis: a biologic link with articular cartilage leading to abnormal remodeling. Curr Opin Rheumatol 2003, 15:628-633.

27. Reichert JC, Quent VMC, Burke LJ, Stansfield SH, Clements JA, Hutmacher DW: Mineralized human primary osteoblast matrices as a model system to analyse interactions of prostate cancer cells with the bone microenvironment. Biomaterials 2010, 31:7928-7936.
28. Grunert M, Dombrowski C, Sadasivam M, Manton K, Cool SM, Nurcombe V: Isolation of a native osteoblast matrix with a specific affinity for BMP2. J Mol Histol 2007, 38:393-404.

29. Dumas V, Ducharne B, Perrier A, Fournier C, Guignandon A, Thomas M, Peyroche S, Guyomar D, Vico L, Rattner A: Extracellular matrix produced by osteoblasts cultured under low-magnitude, high-frequency stimulation is favourable to osteogenic differentiation of mesenchymal stem cells. Calcif Tissue Int 2010, 87:351-364.

30. Sanchez C, Deberg MA, Bellahcene A, Castronovo V, Msika P, Delcour JP, Crielaard JM, Henrotin YE: Phenotypic characterization of osteoblasts from the sclerotic zones of osteoarthritic subchondral bone. Arthritis Rheum 2008, 58:442-455.

31. Batra N, Burra S, Siller-Jackson AJ, Gu S, Xia X, Weber GF, DeSimone D, Bonewald LF, Lafer EM, Sprague E, Schwartz MA, Jiang JX: Mechanical stress-activated integrin alpha5beta1 induces opening of connexin 43 hemichannels. Proc Natl Acad Sci USA 2012, 109:3359-3364.

32. Nishimoto SK, Nishimoto M: Matrix Gla protein C-terminal region binds to vitronectin. Co-localization suggests binding occurs during tissue development. Matrix Biol 2005, 24:353-361.

33. Couchourel D, Aubry I, Delalandre A, Lavigne M, Martel-Pelletier J, Pelletier JP, Lajeunesse D: Altered mineralization of human osteoarthritic osteoblasts is attributable to abnormal type I collagen production. Arthritis Rheum 2009, 60:1438-1450.

34. Bailey AJ, Sims TJ, Knott L: Phenotypic expression of osteoblast collagen in osteoarthritic bone: production of type I homotrimer. Int J Biochem Cell Biol 2002, 34:176-182.

35. Cantatore FP, Corrado A, Grano M, Quarta L, Colucci S, Melillo N: Osteocalcin synthesis by human osteoblasts from normal and osteoarthritic bone after vitamin D3 stimulation. Clin Rheumatol 2004, 23:490-495.

36. Atkins GJ, Rowe PS, Lim HP, Welldon KJ, Ormsby R, Wijenayaka AR, Zelenchuk L, Evdokiou A, Findlay DM: Sclerostin is a locally acting regulator of late-osteoblast/preosteocyte differentiation and regulates mineralization through a MEPE-ASARM-dependent mechanism. J Bone Miner Res 2011, 26:1425-1436.

37. Kalajzic I, Braut A, Guo D, Jiang X, Kronenberg MS, Mina M, Harris MA, Harris SE, Rowe DW: Dentin matrix protein 1 expression during osteoblastic differentiation, generation of an osteocyte GFP-transgene. Bone 2004, 35:74-82.

38. Woo SM, Rosser J, Dusevich V, Kalajzic I, Bonewald LF: Cell line IDG-SW3 replicates osteoblast-to-late-osteocyte differentiation in vitro and accelerates bone formation in vivo. J Bone Miner Res 2011, 26:2634-2646.

39. Qiu S, Sudhaker Rao D, Fyhrie DP, Palnitkar S, Parfitt AM: The morphological association between microcracks and osteocyte lacunae in human cortical bone. Bone 2005, 37:10-15.

40. Globus RK, Amblard D, Nishimura Y, Iwaniec UT, Kim JB, Almeida EA, Damsky CD, Wronski TJ, van der Meulen MC: Skeletal phenotype of growing transgenic mice that express a function-perturbing form of beta1 integrin in osteoblasts. Calcif Tissue Int 2005, 76:39-49.

41. Chen C, Sheppard D: Identification and molecular characterization of multiple phenotypes in integrin knockout mice. Methods Enzymol 2007, 426:291-305.

\section{doi:10.1186/ar4333}

Cite this article as: Prasadam et al: Impact of extracellular matrix derived from osteoarthritis subchondral bone osteoblasts on osteocytes: role of integrin $\beta 1$ and focal adhesion kinase signaling cues. Arthritis Research \& Therapy 2013 15:R150. 